\title{
Duality on hypermaps with symmetric or alternating monodromy group
}

\author{
Daniel Pinto * \\ CMUC, Department of Mathematics, University of Coimbra \\ 3001-454 Coimbra, Portugal
}

Received 23 March 2011, accepted 23 January 2012, published online 22 March 2012

\begin{abstract}
Duality is the operation that interchanges hypervertices and hyperfaces on oriented hypermaps. The duality index measures how far a hypermap is from being self-dual. We say that an oriented regular hypermap has duality-type $\{l, n\}$ if $l$ is the valency of its vertices and $n$ is the valency of its faces. Here, we study some properties of the duality index of oriented regular hypermaps and we prove that for each pair $n, l \in \mathbb{N}$, with $n, l \geq 2$ (but not both equal to 2), it is possible to find an oriented regular hypermap with extreme duality index and of duality-type $\{l, n\}$, even if we are restricted to hypermaps with alternating or symmetric monodromy group.
\end{abstract}

Keywords: Hypermaps, duality, primitive groups, alternating group, symmetric group, generators.

Math. Subj. Class.: 05C10, 05C25, 20B15, 20B35, 20F05

\section{Introduction}

One can look at a hypergraph as a generalization of the well known notion of a graph, since an important restriction is removed: in a hypergraph, an (hyper)edge might connect more than two (hyper)vertices. In a similar way, a hypermap is an obvious generalization of the concept of a map. Although the word map is often used in mathematics with a different meaning, here it is defined as a cellular embedding of a connected graph on a compact connected surface. Without surprise, it is somehow natural to say that a hypermap is, in its topological form, a cellular embedding of a connected hypergraph. Or, alternatively, one can look at a hypermap as an embedding of a connected trivalent graph on a compact connected surface, labelling each face 0,1 or 2 , so that each edge of the trivalent graph is incident to two faces carrying different labels. In this representation of the hypermap (James

* Research supported by CMUC and FCT (Portugal), through European program COMPETE/FEDER.

E-mail address: dpinto@mat.uc.pt (Daniel Pinto) 
representation [9]), the hypervertices are usually represented by label 0 , the hyperedges by label 1 , and the hyperfaces by label 2 . We should note that, by doing this, each hyperedge of the hypermap might be adjacent to more than two hypervertices and, consequently, have valency greater than 2 (something that does not occur in a map). If the valencies of all hyperfaces are equal, and the same happens with the hypervertices and with the hyperedges, we say that the hypermap is uniform. Here, all the surfaces will be orientable and without boundary. It follows that every hypermap can give rise to two oriented hypermaps, one for each fixed orientation. If there is an automorphism that sends one of those oriented hypermaps into the other, we say that the hypermap is reflexible. Otherwise, we call it chiral. The topological idea of an oriented hypermap (a cellular embedding of a hypergraph on an oriented surface), briefly sketched here, has a very useful algebraic translation. In fact, one can look at the results of this paper as purely algebraic results, although they certainly have an important topological meaning.

A hypermap can be regarded as a transitive permutation representation $\Delta \rightarrow \operatorname{Sym} \Omega$ of the group

$$
\Delta=\left\langle r_{0}, r_{1}, r_{2} \mid r_{0}^{2}=r_{1}^{2}=r_{2}^{2}=1\right\rangle \cong C_{2} * C_{2} * C_{2},
$$

on a set $\Omega$ representing its hyperflags (the cells of the barycentric subdivision of the hypermap). Similarly, an oriented hypermap (without boundary) can be regarded as a transitive permutation representation of the subgroup

$$
\Delta^{+}=\left\langle\rho_{0}, \rho_{1}, \rho_{2} \mid \rho_{0} \rho_{1} \rho_{2}=1\right\rangle=\left\langle\rho_{0}, \rho_{2} \mid-\right\rangle
$$

of index 2 in $\Delta$ (a free group of rank 2 ) consisting of the elements of even word-length in the generators $r_{i}$, where $\rho_{0}=r_{1} r_{2}, \rho_{1}=r_{2} r_{0}$ and $\rho_{2}=r_{0} r_{1}$. In the case of hypermaps, the hypervertices, hyperedges and hyperfaces ( $i$-dimensional constituents for $i=0,1,2$ ) are the orbits of the dihedral subgroups $\left\langle r_{1}, r_{2}\right\rangle,\left\langle r_{2}, r_{0}\right\rangle$ and $\left\langle r_{0}, r_{1}\right\rangle$, and in the case of oriented hypermaps they are the orbits of the cyclic subgroups $\left\langle\rho_{0}\right\rangle,\left\langle\rho_{1}\right\rangle$ and $\left\langle\rho_{2}\right\rangle$, with incidence given by nonempty intersection in each case. The local orientation around each hypervertex, hyperedge or hyperface is determined by the cyclic order of the corresponding cycle of $\rho_{0}, \rho_{1}$ or $\rho_{2}$.

An oriented hypermap is regular if it has the highest degree of symmetry. It is well known that, algebraically, an oriented regular hypermap $\mathcal{H}$ can be represented by a triple $(G, x, y)$, where the group $G$, a quotient of $\Delta^{+}$, is generated by the elements $x$ and $y$. If we want to look at those generators from a topological point of view, $x$ can be interpreted as the permutation that cyclically permutes the hyperdarts (oriented hyperedges) based on the same hypervertex, and $y$ the permutation that cyclically permutes the hyperdarts based on the same hyperface, according to the chosen orientation. It is not difficult to verify that every regular hypermap is uniform (in the James representation: every face labelled $i$, with $i \in\{0,1,2\}$, has the same valency). We say that a regular hypermap has type $(l, m, n)$ (for $l, m, n \in \mathbb{N}$ ) if $l, m$ and $n$ are the valencies of its hypervertices, hyperedges and hyperfaces, respectively. The group $G=\langle x, y\rangle$ is called the monodromy group of the hypermap (and, since the hypermap is regular, it coincides with its automorphism group). Duality, one of the many possible operations on hypermaps (see [8]), is an operation that interchanges hyperfaces and hypervertices, which in terms of the generators of the monodromy group is the same as saying that the dual of $\mathcal{H}=(G, a, b)$ is $\mathcal{H}^{d}=(G, b, a)$. If there is an automorphism of $G$ that interchanges $a$ and $b$, we say that $\mathcal{H}$ is self-dual (invariant under duality) and $\mathcal{H} \cong \mathcal{H}^{d}$. 


\section{Duality index}

A possible way to define the duality group of a hypermap $\mathcal{H}=(G, x, y)$, as an extension of the notion of chirality group (see [1], [8]), is to say that it is the minimal subgroup $D(\mathcal{H}) \unlhd G$ such that the quotient hypermap

$$
\mathcal{H} / D(\mathcal{H})=(G / D(\mathcal{H}), x D(\mathcal{H}), y D(\mathcal{H}))
$$

is a self-dual hypermap. The order of this duality group is called the duality index $d$ of $\mathcal{H}$, and it is a measure of how far a hypermap is from being self-dual. If the duality index is 1 , then the hypermap is self-dual; and the bigger that index, the more distant the hypermap is from being self-dual. If $D(\mathcal{H}) \cong G$, the duality group is isomorphic to the monodromy group of the hypermap and we say that the hypermap has extreme duality index.

\section{The symmetric and the alternating groups}

Before dealing with more difficult problems, we start this section by proving a simple lemma:

Lemma 3.1. For every $n \in \mathbb{N}$ :

a) there is a non-self-dual hypermap with monodromy group $S_{n}$.

b) there is a non-self-dual hypermap with monodromy group $A_{n}$.

Proof. a) If $n>2$, we take $x=(1,2, \ldots, n)$ and $y=(1,2)$. These permutations generate the group $S_{n}$ but, because they have different orders, there is no automorphism that interchanges those two generators. It follows that the hypermap $\mathcal{H}=\left(S_{n}, x, y\right)$ is not self-dual. If $n=2, S_{2} \cong C_{2}=\langle g\rangle$ and $\left(C_{2}, 1, g\right)$ is not self-dual (if we take 1 as the neutral element of $C_{2}$ ).

b) For $n>3$, we just need to apply that same idea but using the canonical generators $x=(1,2, \ldots, n)$ and $y=(1,2,3)$, if $n$ is odd, or $x=(2,3, . ., n)$ and $y=(1,2,3)$, if $n$ is even. Because, in both cases, the two generators do not have the same order, the hypermap is not self-dual. If $n=3, A_{n}=A_{3} \cong C_{3}=\langle g\rangle$ and $\left(C_{3}, 1, g\right)$ is not self-dual.

Example 3.2. Let $G=A_{5}=\langle x, y\rangle$, where $x=(1,2,3,4,5)$ and $y=(1,2,3)$. Because $A_{5}$ is simple and the hypermap $\left(A_{5}, x, y\right)$ is not self-dual, the duality group (which is normal in $A_{5}$ ) must be $A_{5}$ itself. Hence, the duality index of the hypermap is $\left|A_{5}\right|=5 ! / 2$.

More generally, if we take

$$
\left(A_{n},(1,2, \ldots, n),(1,2,3)\right) \text {, if } n \text { is odd, or }\left(A_{n},(2,3, \ldots, n),(1,2,3)\right) \text {, if } n \text { is even, }
$$

we get hypermaps with extreme duality index and with monodromy group $A_{n}$. Hence:

Theorem 3.3. If $n \geq 3$ there is a hypermap with extreme duality index and with monodromy group $A_{n}$. 
Remarks: a) Since each one of those hypermaps $\mathcal{H}$ has extreme duality index, $D(\mathcal{H})=$ $\operatorname{Mon}(\mathcal{H})=A_{n}$. It follows that, for any $n \geq 3, A_{n}$ is the duality group of some hypermap. b) If we take any simple group generated by two elements of different orders, we can get a similar result.

If $G=S_{n}$, the only possible duality groups are $1, A_{n}$ and $S_{n}$. Therefore, a hypermap with monodromy group $S_{n}$ is self-dual, has extreme duality index or has $n ! / 2$ as duality index. For $n \neq 6$, all automorphisms of $S_{n}$ are inner and act by conjugation. If there is an automorphism that transposes the two generators, the hypermap is self-dual and then $\mathcal{D}(\mathcal{H})=1$. Otherwise, $\mathcal{D}(\mathcal{H})=A_{n}$ or $S_{n}$ and we need to check if the hypermap with monodromy group $S_{n} / A_{n}$, of order 2 , is self-dual or not.

Theorem 3.4. Every hypermap $\mathcal{H}=\left(S_{n}, x, y\right)$ :

i) has extreme duality index if $x$ or $y$ is an even permutation;

ii) is self-dual or has duality index $n ! / 2$ if $x$ and $y$ are both odd permutations and $n \neq 4$.

iii) is self dual or has duality index 4 if $x$ and $y$ are both odd permutations and $n=4$.

Proof. i) The only non-identity quotients $S_{n} / N$ of $S_{n}$ are $S_{n} / A_{n} \cong C_{2}$, and $S_{4} / V_{4} \cong S_{3}$ when $n=4$. In each case, because one of $x N$ and $y N$ is in the unique subgroup of index 2 of $S_{n} / N$ and the other is not, there can be no automorphism of $S_{n} / N$ transposing $x N$ and $y N$. So, the only self-dual quotient is the trivial one and the hypermap has extreme duality index.

ii) $S_{n}$ is not cyclic and, by definition $\langle x, y\rangle=S_{n}$. Hence, $x \neq y$. Suppose $\mathcal{H}$ is not self-dual. Because $\mathcal{H} / A_{n}=\left(S_{n} / A_{n}, x A_{n}, y A_{n}\right)$ and $\left|S_{n} / A_{n}\right|=2$, the two generators $x A_{n}$ and $y A_{n}$ must be the same. It follows that the hypermap $\mathcal{H} / A_{n}$ is self-dual and $|D(\mathcal{H})|=n ! / 2$.

iii) Let $x$ and $y$ be two odd permutations generating $S_{4}$ and $N$ the Klein group $V_{4}$ (a normal subgroup in $S_{4}$ ). That pair of generators may be formed by a transposition and a 4-cycle or by two 4-cycles. Say $x$ is a transposition and $y$ is a 4-cycle. Then, they map to distinct permutations in $S_{4} / N \cong S_{3}$. Because the third element in $S_{3}$ conjugates each to the other, the quotient hypermap is self-dual (whereas the hypermap itself is not), with duality group $V_{4}$. If $x$ and $y$ are both 4-cycles the hypermap is self-dual.

Can we have an oriented regular hypermap with extreme duality index for each type $(l, m, n)$ ?

The answer is no. There are no hypermaps of extreme duality index and of type $(3,2,3)$, since this has to be the tetrahedron, which is self-dual. On the other hand, if we restrict ourselves to hyperbolic triples, where $\frac{1}{l}+\frac{1}{m}+\frac{1}{n}<1$, we can enumerate orientably regular hypermaps of a given type $(l, m, n)$ with automorphism groups isomorphic to $\operatorname{PSL}(2, q)$ or $P G L(2, q)$ (this enumeration can be found in a joint work of Marston Conder, Primoz Potocnik and Josef Siran [6], based on a paper by Sah [13]). Because these groups are simple or almost simple, we can use them to try to find hypermaps with extreme duality index or self-dual hypermaps. If $l \neq n$ the hypermap cannot be self-dual. If $l=n$, we have to check if there is an automorphism of $\operatorname{PSL}(2, q)$ or $\operatorname{PGL}(2, q)$ that interchanges the two generators. 
We should also notice that for some triples $(l, m, n)$ is very easy to find hypermaps with extreme duality index and of that type.

If $\operatorname{gcd}(l, n)=1$ then

$$
G_{l, m, n}=\left\langle a, b \mid a^{l}=b^{n}=(a b)^{m}=1\right\rangle
$$

is obviously the monodromy group of a hypermap of type $(l, m, n)$. Let $N$ be the duality group of the hypermap $\left(G_{l, m, n}, a, b\right)$. Then $N$ is the smallest normal subgroup of $G_{l, m, n}$ such that $G_{l, m, n} / N$ is reflexible, which means that we are working with the smallest normal subgroup $N$ of $G_{l, m, n}$ such that the assignment $a \mapsto b, b \mapsto a$ induces an automorphism of $G_{l, m, n} / N$. We obtain this quotient by adding extra relations, substituting $a$ for $b$ and $b$ for $a$ in the original ones. Then:

$$
G_{l, m, n} / N=\left\langle a, b \mid a^{l}=a^{n}=b^{n}=b^{l}=(a b)^{m}=(b a)^{m}=1\right\rangle .
$$

Because $l$ and $n$ are co-prime, the group $G_{l, m, n} / N$ collapses to the identity. Therefore, $\mathcal{H}=\left(G_{l, m, n}, a, b\right)$ is a hypermap with extreme duality index and of type $(l, m, n)$. This proves that, if $\operatorname{gcd}(l, n)=1$, for every triple $(l, m, n)$ there is a hypermap with extreme duality index and of that type $(l, m, n)$.

\subsection{Alternating monodromy group}

In the 1960's, Graham Higman conjectured that any Fuchsian group has among its homomorphic images all but finitely many of the alternating groups. He also proved that $A_{n}$ is a factor group of $(2,3,7)=\left\langle a, b \mid a^{2}, b^{3},(a b)^{7}\right\rangle$ for all large $n$. Because 2, 3 and 7 are distinct prime numbers, we can conclude, from that result, that there is an infinite number of hypermaps with extreme duality index and of type $(2,3,7)$. The result obtained by Higman was later extended by others (Marston Conder [5], for instance) that proved that the same can be said for other families of triangle groups. The complete proof of the conjecture was published in 2000 by Brent Everitt [7]. In his paper, it is shown that we need only consider the triangle groups $(p, q, r), 3 \leq p<q<r$, to prove the main result (which is done with the help of coset diagrams for those triangle groups). Hence, it is possible to say that if $l, m, n$ are prime and $l \neq n$ we can always find infinite hypermaps with extreme duality index and of type $(l, m, n)$, with alternating group as monodromy group. If $p, q, r$ are not all prime, then the alternating groups, being factor groups of the triangle group $(p, q, r)$, might correspond to hypermaps of type $\left(p^{\prime}, q^{\prime}, r^{\prime}\right)$ with $p^{\prime}\left|p, q^{\prime}\right| q$ and $r^{\prime} \mid r$, and not always of type $(p, q, r)$. It follows that we cannot directly apply the result of Brent Everitt to prove that there are hypermaps of any type with alternating monodromy group. The proof of Brent Everitt would had to be changed, in order to sustain that conclusion. Instead, we take a different approach.

\subsection{Duality-type of hypermaps with symmetric or alternating monodromy group}

Definition 3.5. We say that an oriented regular hypermap has duality-type $\{l, n\}$ if $l$ is the valency of its vertices and $n$ is the valency of its faces (which is the same as saying that $l$ and $n$ are the orders of the generators interchanged by the duality operation).

If we restrict ourselves to the family of hypermaps whose monodromy group is the alternating or the symmetric group, can we have a hypermap with extreme duality index and 
of any duality-type $\{l, n\}$ ? In fact, we can not only prove that the answer is affirmative (if $n, l \geq 2$ but not both equal to 2 ) but also explicitly show how to construct such hypermaps. The reason why, here, we look for duality-type instead of type is because, in the first case, we just need to control the order of the two generators $x$ and $y$, while in the second case we also need to pay attention to the order of $x y$, which seems a harder problem to solve. To complete that easier first task, we still need to add a few concepts and theorems about primitive groups.

Let $(G, \Omega)$ be a permutation group. An equivalence relation $\sim$ is called $G$-invariant if whenever $\alpha, \beta \in \Omega$ satisfy $\alpha \sim \beta$ then $g(\alpha) \sim g(\beta)$ for all $\alpha, \beta \in \Omega$. Two obvious $G$-invariant equivalence relations are: (i) $\alpha \sim \beta$ if and only if $\alpha=\beta$ and (ii) $\alpha \sim \beta$ for all $\alpha, \beta \in \Omega$. We call $(G, \Omega)$ imprimitive if it admits some equivalence relation other than (i) or (ii). Otherwise, we call $(G, \Omega)$ primitive.

There are several examples of primitive groups (Colva M. Roney-Dougal [11] has classified all the primitive permutation groups of degree less than 2500). For instance, any alternating group $A_{n}$ is primitive, and so is $\operatorname{PGL}(2, q)$, in its standard action, for any prime power $q$. However, it is known - as underlined by Peter Cameron in the Encyclopedia of Design Theory [3] - that primitive groups are "rare (for almost all $n$, the only primitive groups of degree $n$ are the symmetric and alternating groups, see [4]); and small (of order at most $n^{c \cdot \log n}$ with known exceptions)". The fact that most of the primitive groups are alternating groups (which are simple) and symmetric groups (which have only one non trivial normal subgroup) makes primitivity a powerful concept to build hypermaps with extreme duality index. Although the probability of a primitive group being an alternating or a symmetric group is very high, we need to be sure that we are not dealing with a different kind of group. The next definitions and theorems help us to achieve that goal.

Definition 3.6. Let $G$ be a permutation group on $\Omega$ and $k$ a natural number with $1 \leq k \leq$ $n=|\Omega|$. $G$ is called $k$-transitive if for every two ordered $k$-tuples $\alpha_{1}, \ldots \alpha_{k}$ and $\beta_{1}, \ldots, \beta_{k}$ of points of $\Omega$ (with $\alpha_{i} \neq \alpha_{j}, \beta_{i} \neq \beta_{j}$, for $i \neq j$ ) there exists $g \in G$ which takes $\alpha_{t}$ into $\beta_{t}($ for $t=1, \ldots, k)$.

Definition 3.7. The degree of a permutation group is the number of points moved by at least one of its permutations.

Definition 3.8. The minimal degree of a permutation group is the minimum number of points moved by any non-identity element of the group.

Theorem 3.9. [10] The minimal degree of a primitive group which is neither alternating nor symmetric must exceed 4 whenever its degree exceeds 8.

Theorem 3.10. [14] A primitive group of degree $n$, which contains a cycle of degree $m$ with $1<m<n$ is $(n-m+1)$-transitive.

Theorem 3.11. [12] A 2-transitive permutation group is primitive.

And, as a consequence of the classification of simple groups [2]:

Theorem 3.12. If a permutation group $G$ is at least 6 -transitive then $G$ is the alternating group or the symmetric group. 
With these tools we can now prove the following theorem:

Theorem 3.13. For each pair $n, l \in \mathbb{N}$, with $n, l \geq 2$ (but not both equal to 2 ), it is possible to find an oriented regular hypermap with extreme duality index and of duality-type $\{l, n\}$, with alternating or symmetric group as monodromy group.

Proof. The general idea behind this proof is the following: first, we try to prove that the two chosen permutations generate a primitive group, and then we show that they must generate the symmetric or the alternating group. If they generate the symmetric group (which is not a simple group), we also need to check that they give rise to a hypermap with extreme duality index.

a) If $l, n$ are not both even and $l \neq n$ (we may assume, without loss of generality, that $l>n)$, let $x=(1,2, \ldots, l)$ and $y=(1,2, \ldots, n)$ be two permutations of $S_{l}$.

Then, since the stabilizer of the point $l$ contains $y$ and its conjugates under suitable powers of $x$, the group $G=\langle x, y\rangle$ is 2-transitive. Hence, by Theorem 3.11, $G$ is primitive.

If we now take $z$ as the commutator $y^{-1} x^{-1} y x=(n, n-1, l)$, we can, by Theorem 3.9 , conclude that if $l>8, \mathrm{G}$ must be the alternating or the symmetric group. If $l$ and $n$ are both odd, $G$ must be the alternating group and $\mathcal{H}=\left(G=A_{l}, x, y\right)$ is a hypermap of extreme duality index and of duality type $\{l, n\}$. If $l$ is even and $n$ is odd (or if $l$ is odd and $n$ is even), $G=\langle x, y\rangle=S_{l}$. Because not both of the generators are odd permutations, $\mathcal{H}=(G, x, y)$ has extreme duality index (see Theorem 3.4).

(For $l<8$ we can easily find two permutations, one of order $l$ and another of order $n$, that generate $A_{l}$, when both permutations are odd, and $S_{l}$, in the other cases).

b) Suppose $n=l$ (both odd).

Let the generators of the group be these two even permutations in $S_{l+1}$ :

$$
\begin{gathered}
x=(1,2, \ldots, l) \\
y=(2,3, \ldots, l+1) .
\end{gathered}
$$

The group generated by these two elements is primitive since it is a 2-transitive group (Theorem 3.11). Moreover, $x^{-1} y^{-1} x y=(1, l+1)(l-1, l)$ is a permutation that just moves four points. It follows that $G=\langle x, y\rangle$ is a group of minimal degree $\leq 4$. Hence, by Theorem 3.9, $\langle x, y\rangle=A_{l}$ if $l+1 \geq 8$, i.e. $l \geq 7$. Cases for small $l$ are easy to solve.

c) If $l$ and $n$ are both even, we assume (without loss of generality) that $l \geq n$. We then take:

$$
\begin{gathered}
x=(1,2, \ldots, l) \\
y=(1,2)(l, l+1, l+2, \ldots, l+n-1)
\end{gathered}
$$

permutations of $S_{l+n-1}$ and $G=\langle x, y\rangle$. G is clearly a transitive group. Because $x y=(1,3,4, \ldots, l-1, l, l+1, l+2, \ldots, l+n-1)$ we can conclude that the stabilizer of the point 2 acts transitively on the remaining points. Therefore, $G=\langle x, y\rangle$ is a 2 -transitive group and, by Theorem $3.11, G$ is also a primitive group of degree $l+n-1$. 
Since $x$ is a cycle of order $l$ and $l+n-1-l+1=n$, the group $G$ is $n$-transitive (Theorem 3.10). For $n>5, G$ must be the alternating or symmetric group (Theorem 3.12). If $n=4$ or $n=2$ the degree of $y^{2}$ does not exceed four and the group must be the alternating or the symmetric group (Theorem 3.9). Since $x$ is an odd permutation, $G=S_{l+n-1}$. Then $\mathcal{H}=\left(S_{l+n-1}, x, y\right)$ is a hypermap with extreme duality index because $y$ is an even permutation (see Theorem 3.4).

Note: Because two involutions must always generate a dihedral group, no alternating group can be generated by two involutions, and the only symmetric group with that property is $S_{3}$. But if an oriented hypermap has monodromy group $S_{3}$ and is generated by two involutions, it must be self-dual. Therefore, it is not possible to find an oriented regular hypermap with extreme duality index and of duality-type $\{2,2\}$ with alternating or symmetric group as monodromy group.

With the proof of the previous theorem, we have not only shown that it is possible to find the required hypermaps, but also to describe them, since the generators of the alternating or symmetric groups are explicitly given for each case. We have also proved a slightly weaker result:

Theorem 3.14. For any $l, n \in \mathbb{N}$, with $n, l \geq 2$, one can find two permutations $x$ and $y$ (of orders $l$ and $n$, respectively), such that $x$ and $y$ generate an alternating or a symmetric group.

\section{References}

[1] A. Breda D’Azevedo, G. Jones, R. Nedela and M. Škoviera, Chirality Groups of Maps and Hypermaps, J. Algebraic Combin. 29 (2009), 337-355.

[2] P. J. Cameron, Finite permutation groups and finite simple groups, Bull. London Math. Soc. 13 (1980), 1-22.

[3] P. J. Cameron, The Encyclopaedia of Design Theory (Primitive permutation groups, http: //designtheory.org/library/encyc/).

[4] P. J. Cameron, Peter M. Neumann and D. N. Teague, On the degrees of primitive permutation groups, Math. Z 180 (1982), 141-149.

[5] M. Conder, More on generators for alternating and symmetric groups, Quart. J. Math. Oxford 32 (1981), 137-163.

[6] M. Conder, P. Potočnik and J. Širáň, Regular hypermaps over projective linear groups, J. Aust. Math. Soc. 85 (2008), 155-175.

[7] B. Everitt, Alternating Quotients of Fuchsian Groups, J. Algebra 223 (2000), 457-476.

[8] G. A. Jones and Daniel Pinto, Hypermap operations of finite order, Discrete Math. 310 (2010), $1820-1827$.

[9] Lynne D. James, Representations of Maps, Disertaciones del Seminario de Matemáticas Fundamentales, 8. Universidade Nacional de Educatión a Distancia, Madrid, 1990.

[10] G. A. Miller, Possible Orders of two generators of the alternating and of the symmetric group. Trans. Amer.Math. Soc. 30 (1928), 24-32. 
[11] C. M. Roney-Dougal, The primitive groups of degree less than 2500, J. Algebra 292 (2005), 154-183.

[12] J. Rotman, Introduction to the Theory of Groups, 4th edition, Springer, 1999.

[13] C. M. Sah, Groups related to compact Riemann surfaces, Acta Math. 123 (1969), 13-42.

[14] H. Wielandt, Finite Permutation Groups, 1st edition, Academic Press, 1964. 